12,03

\title{
Поляризационная спектроскопия одиночной квантовой точки и одиночной квантовой нити
}

\author{
() В.Н. Кац ${ }^{1}$, А.В. Платонов ${ }^{1}$, Г.Э. Цырлин ${ }^{1}$, А.Д. Буравлев ${ }^{1}$, A. Delga ${ }^{2}$, \\ L. Besombes ${ }^{2}$, H. Mariette $^{2}$, В.П. Кочерешко ${ }^{1}$ \\ ${ }^{1}$ Физико-технический институт им. А.Ф. Иоффре РАН, \\ Санкт-Петербург, Россия \\ ${ }^{2}$ Institute Neel, \\ Grenoble, France \\ E-mail: Vladimir.N.Kats@gmail.com \\ (Поступила в Редакцию 22 мая 2018 г.)
}

Исследованы спектры фотолюминесценции одиночной квантовой точки GaAs, заключенной в квантовую нить AlGaAs. Из поведения спектров в магнитном поле удалось оценить энергию связи экситона в квантовой точке, которая оказалась в 10 раз больше энергии связи объемного экситона в GaAs. Обнаружено, что сигнал экситонной фотолюминесценции из квантовой точки, излучаемый в направлении оси нанонити, поляризован линейно. В то же время, сигнал фотолюминесценции, распространяющийся в направлении, перпендикулярном оси нанонити, практически не поляризован. Наблюдаемый эффект может быть объяснен неаксиальным расположением точки относительно оси нити в условиях гигантского увеличения энергии связи экситона, связанного с влиянием на экситон потенциала изображения.

В.П. Кочерешко благодарит программу отделения физических наук РАН „Электронные корреляции в системах с сильным взаимодействием“. Работа А.В. Платонова была поддержана Программой Президиума РАН № 9 „Терагерцовая оптоэлектроника и спинтроника“.

DOI: 10.21883/FTT.2018.12.46737.136

\section{1. Введение}

Технология молекулярно-лучевой эпитаксии (МЛЭ) с использованием катализатора в виде металлических капель на поверхности, разработанная в последние годы, позволяет создавать квантовые нити или нанонити $(\mathrm{HH})$ длиной до $10 \mu \mathrm{m}$ и диаметром 20-200 nm. В процессе роста таких нитей можно варьировать их состав, что позволяет получать квантовые точки (КТ), встроенные в нить [1].

Эти объекты имеют ряд уникальных свойств. Прежде всего, можно создать КТ с точно определенным размером, поэтому положение энергетических уровней в КТ хорошо контролируется [2]. Во-вторых, достаточно точно контролируется расстояние между несколькими точками внутри одного провода, что позволяет создавать цепочки (две или более) КТ с предопределенным туннельным взаимодействием между соседним и КТ. Втретьих, можно создавать упорядоченные массивы НН. Наконец, поскольку сама нить является макроскопическим объектом, можно создать контакты, которые позволяют получить токовую накачку одной точки. Это делает систему чрезвычайно перспективной как для фундаментальных, так и прикладных исследований.

\section{2. Эксперимент}

В работе исследованы нанонити $\mathrm{AlGaAs}$, содержащие GaAs квантовые точки. Структуры выращивались на полуизолирующих подложках $\mathrm{GaAs}$ [111] в установке MBE EP1203, снабженной твердым источником Ga и Al и дополнительными ячейками мышьяка. Использовалась, уже ставшая стандартной, каталитическая методика роста квантовых нитей [3]. В используемой ориентации подложки НН растут строго перпендикулярно поверхности.

Характеризация образцов проводилась с использованием сканирующего электронного микроскопа (SEM) и просвечивающего электронного микроскопа (ТЕM). Диаметр НН в наших образцах составлял 20-50 nm, длина НН достигала 500-1000 nm, толщина КТ составляла $2-5 \mathrm{~nm}$.

Спектры фотолюминесценции (ФЛ) при непрерывном и импульсном возбуждении измерялись при температурах от 5 до $250 \dot{К}$ в зависимости от интенсивности оптического возбуждения и внешнего магнитного поля. Для регистрации спектров использовался монохроматор Jobin-Yvon $50 \mathrm{~cm}$ и CCD детектор. Спектры микрофотолюминесценции одиночной нанонити измерялись с помощью микроскопа. Минимальный размер лазерного пятна на образце составлял около $1.5 \mu \mathrm{m}$, что позволило нам выделять сигнал от одиночной нити при плотности нитей $10^{-11} \mathrm{~cm}^{-2}$. Изучались одиночные нанонити (HH) $\mathrm{Al}_{0.26} \mathrm{Ga}_{0.74} \mathrm{As}$ диаметром 20-25 nm, длиной $1 \mu \mathrm{m}$, внутри нанонити была выращена квантовая точка (КТ) толщиной 2.5-3.0 nm и диаметром 15-20 nm.

На рис. 1 представлен спектр ФЛ одиночной нанонити при очень маленькой интенсивности оптическо- 


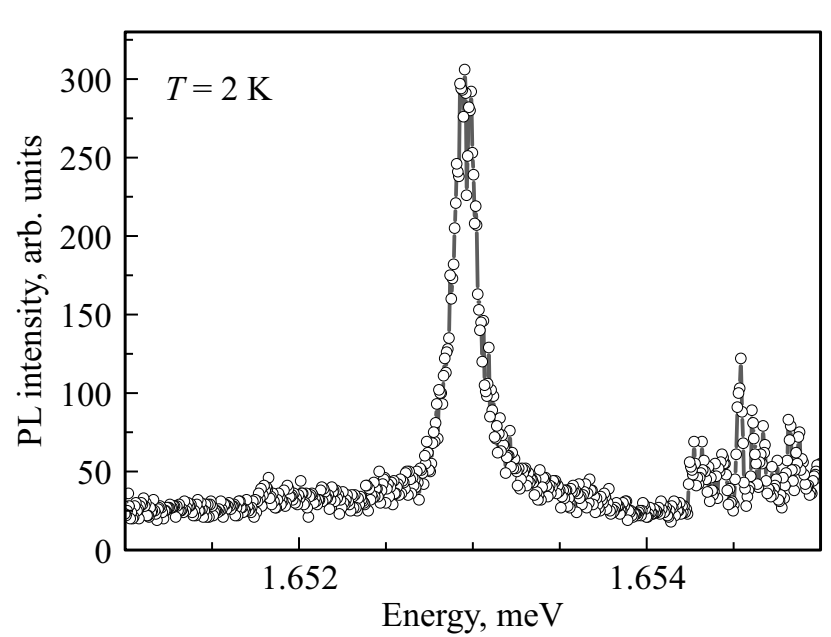

Рис. 1. Спектр фотолюминесценции экситона из одиночной квантовой точки GaAs, заключенной в квантовую нить $\mathrm{Al}_{0.26} \mathrm{Ga}_{0.74} \mathrm{As}$. Толщина точки $2.5 \mathrm{~nm}$, диаметр $15 \mathrm{~nm}$.

го возбуждения, не превышающей $0.1 \mathrm{~W} / \mathrm{cm}^{2}$. В спектре наблюдается узкая, симметричная линия, шириной $0.2 \mathrm{meV}$, связанная с рекомбинацией экситона в одиночной квантовой точке, заключенной в нанонить. При увеличении интенсивности фото-возбуждения положение этой линии в спектре менялось скачками в одну и/или другую сторону. Частота этих скачков росла по мере увеличения интенсивности возбуждения. В результате эта линия ФЛ регистрировалась фотодетектором как очень широкая линия, шириной порядка $1-2 \mathrm{meV}$.

Так как мы имеем дело с одиночным нанообъектом, то никакого неоднородного уширения линий тут быть не может. Большая ширина линии ФЛ экситона при достаточно интенсивном фотовозбуждении объяснялась эффектом динамического уширения [4]. Положение линии ФЛ в НН достаточно малого диаметра, когда экситон находится в непосредственной близости от поверхности, зависит от наличия зарядов на поверхности нити. В условиях относительно сильного оптического возбуждения распределение зарядов на поверхности нити меняется, что и проявляется в виде скачков положения линии.

Для определения параметров экситона в КТ мы провели измерения спектров ФЛ в магнитных полях до 8 Т. На рис. 2 представлена зависимость энергетического положения линии экситонной люминесценции от магнитного поля в правой $\sigma^{+}$и левой $\sigma^{-}$круговых поляризациях.

Из полученных зависимостей мы определили эффективный $g$-фактор экситона, который оказался равным $g \approx 1.0$ [5], и константу диамагнитного сдвига экситона, которая оказалась значительно меньше диамагнитного сдвига экситона в объемном GaAs [6]. Маленькая величина диамагнитного сдвига может указывать на большую энергию связи экситона в КТ, заключенной в НН.

Из зависимости, приведенной на рис. 2, получаем, что константа диамагнитного сдвига экситона в квантовой точке, заключенной в квантовую нить, равна $d \approx 3 \cdot 10^{-3} \mathrm{meV} / \mathrm{T}^{2}$. Как известно, константа диамагнитного сдвига связана с радиусом экситона соотношением [7]

$$
d=\frac{e^{2}}{8 \mu c^{2}} a_{B}^{2},
$$

где $\mu-$ приведенная масса, $c-$ скорость света, $e-$ заряд электрона, $a_{B}-$ боровский радиус экситона в плоскости, перпендикулярной магнитному полю.

Константа диамагнитного сдвига в широких квантовых ямах GaAs (практически объемном материале), шириной $40 \mathrm{~nm} б$ составляет $0.06 \mathrm{meV} / \mathrm{T}^{2}$ [6]. Можно также оценить константу диамагнитного сдвига объемного экситона, пользуясь измеренной нами величиной диамагнитного сдвига объемном CdTe [8], принимая во внимание различие эффективных масс в этих материалах. Получается также величина порядка $0.06 \mathrm{meV} / \mathrm{T}^{2}$. Таким образом, зная боровский радиус экситона в объемном $\mathrm{GaAs}$, получаем, что боровский радиус экситона в плоскости перпендикулярной оси нити, примерно в 4.5 раза меньше его объемного значения и составляет $2.8 \mathrm{~nm}$, что заметно меньше радиуса самой нити.

Если предположить, что экситон в нашей НН имеет сферическую симметрию, то для такого боровского радиуса получим энергию связи $90 \mathrm{meV}$.

В работах [9-11] было показано, что энергия связи экситона в НН может достигать огромных величин, до нескольких сотен $\mathrm{meV}$ и даже единиц $\mathrm{eV}$. Причина такого роста энергии связи экситона заключается в усилении кулоновского взаимодействия в экситоне благодаря диэлектрическому контрасту. Дело в том, что статическая диэлектрическая проницаемость материала нити равна примерно 13, нить окружена средой с диэлектрической проницаемостью, равной 1. Следовательно, во-первых, на заряды внутри нити будут действовать силы от потенциала изображения этих зарядов, стремящиеся

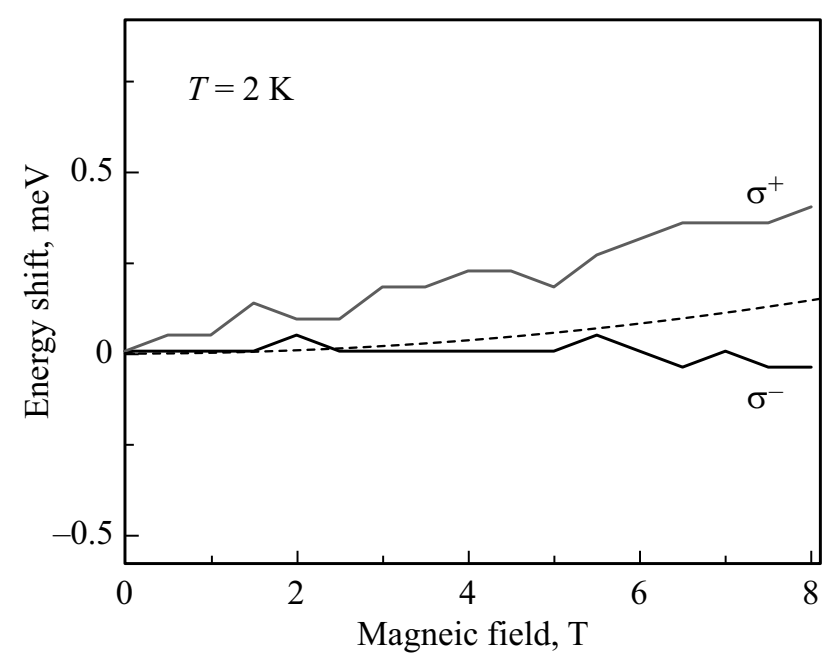

Рис. 2. Зависимость энергетического сдвига линий экситонной ФЛ от образца с НН, содержащего одиночную квантовую точку в магнитном поле, изменяющемся до 8 Т. Пунктиром показана аппроксимация полусуммы двух зеемановских компонент. 
увеличить их конфайнмент. Во-вторых, экранирование кулоновского взаимодействия в экситоне, находящемся внутри НН, будет ослаблено [9-11].

Полный разрыв зон в наших НН составляет порядка $300 \mathrm{meV}$. Тогда из положения линии ФЛ экситона

$$
E_{P L}=E_{g}^{\mathrm{GaAs}}+E_{c}+E_{v}-E_{\text {exc }}
$$

(здесь $E_{c}$ и $E_{v}$ - энергии размерного квантования электрона и дырки, $E_{e x c}$ - энергия связи экситона) можно оценить энергии размерного квантования носителей. Отсюда при $E_{P L}=1.653 \mathrm{meV}$ и $E_{\text {exc }}=90 \mathrm{meV}$ получаем, что суммарная энергия квантования носителей должна быть $224 \mathrm{meV}$. Такая энергия размерного квантования носителей получится для носителей, запертых в квантовой точке толщиной 2.5-3.0 nm, окруженной вдоль оси нити барьерами с суммарной высотой $300 \mathrm{meV}$ и в плоскости бесконечно высокими барьерами.

Таким образом, можно заключить, что экситон, скорее, выглядит как сферический, чем как квазидвумерный. Если взять энергию связи экситона $50 \mathrm{meV}$, то получаем качественно похожий результат.

В спектрах поляризованной фотолюминесценции одиночной нанонити был обнаружен необычный эффект. Было обнаружено, что экситонное излучение в направлении [111] линейно поляризовано в осях [1힐 и перпендикулярной оси $[11 \overline{2}]$. Эти направления совпадают с соответствующими кристаллографичекими осями подложки. Степень линейной поляризации $P_{\text {lin }}$ варьировалась от образца к образцу в диапазоне от 10 до 50\%

$$
P_{l i n}=\frac{I_{[11 \overline{0}]}-I_{[11 \overline{2}]}}{I_{[11 \overline{0}]}+I_{[11 \overline{2}]}}
$$

На рис. 3 представлены спектры поляризованной фотолюминесценции экситона из одиночной КТ, заключенной в НН.

Из рисунка ясно видно, что экситонное излучение квантовой точки вдоль оси проволоки (вдоль направления [111]) — линейно поляризовано. Это выглядит удивительно, потому что ось роста проволок соответствует кристаллографической оси третьего порядка, обладающей высокой симметрией (как минимум $C_{3}$ для обеих возможных кристаллических фаз, вюрцитной и цинковой обманки). Поэтому никакой анизотропии НН в плоскости, перпендикулярной оси [111], в результате чего могла бы появиться линейная поляризация излучения, быть не может. Здесь следует отметить два важных факта: 1) степень поляризации сильно варьируется от образца к образцу и 2) направление поляризации привязано к кристаллографическим осям подложки. Важной особенностью этой линии люминесценции является то, что ее поляризация не связана с расщеплением самой линии люминесценции, как это обычно наблюдалось в анизотропных квантовых точках [12].

Очевидно, что причина линейной поляризации заключается в анизотропии оптических свойств, вызванной понижением симметрии нанонити. Для получения

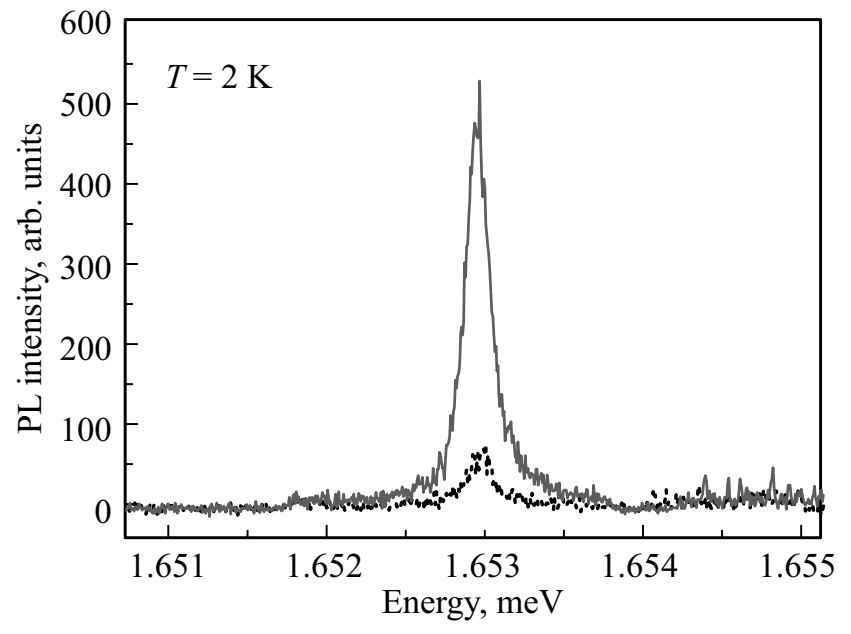

Рис. 3. Спектр линейно поляризованной фотолюминесценции. Сплошная кривая - поляризация вдоль [1ํㅣㄹ, пунктирная $[11 \overline{2}]$.

степени поляризации в 50\% искажение формы нити должно быть довольно большим, также порядка 50\%. Тщательные SEM- и TEM-исследования показывают, что форма нанонити является гексагональной или близкой к гексагональной. Тем не менее, конечно, искажение формы нити перпендикулярно ее оси может быть причиной наблюдаемой линейной поляризации экситонного излучения вдоль оси [111] нити. Другой вероятной причиной появления линейной поляризации может быть не аксиальное расположение квантовой точки внутри нанонити. Других причин этого мы предположить не можем.

Отсутствие расщепления линии ФЛ на компоненты, поляризованные вдоль $[11 \overline{0}]$ и $[11 \overline{2}]$ осей, указывает на то, что главным в возникновении линейной поляризации ФЛ является не анизотропия волновых функций экситона, а анизотропия распределения электромагнитного поля вокруг нити. Анизотропия формы нити неизбежно приводит к анизотропии волновых функций экситона и, следовательно, к расщеплению уровней. Поэтому мы считаем, что неаксиальное расположение точки внутри нити предпочтительнее для объяснения наблюдаемой линейной поляризации.

Как мы только что видели в экспериментах с магнитным полем, влияние диэлектрического контраста на экситон может быть весьма значительным. Следовательно, даже небольшое возмущение электромагнитного поля при смещении точки от центра нити также может быть большим. Оценки показывают, что даже смещение центра точки на 10\% может дать линейную поляризацию излучения порядка $30 \%$.

Из одного только оптического эксперимента нельзя указать причину неаксиального расположения точки внутри нити. Однако мы можем предположить, что это может быть связано с различием скоростей роста в разных кристаллографических направлениях. Такая 

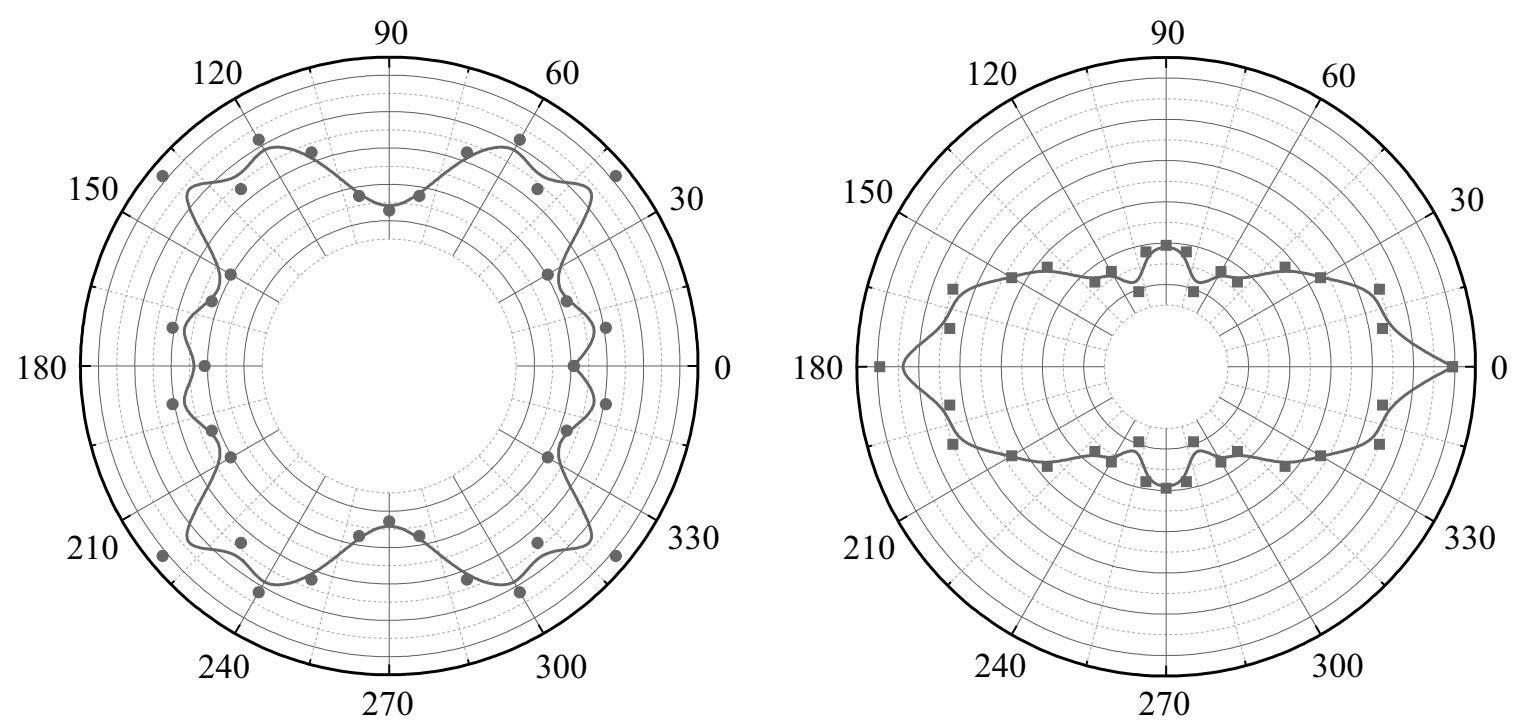

Рис. 4. Диаграмма линейной поляризации излучения из квантовой точки в „лежащей“ квантовой нити.

анизотропия может также возникнуть из-за того, что нити могут иметь смешанную фазу вюрцит-сфалерит [3]. Это может быть связано также и с наклонным расположением источников в камере МЛЭ. Для выяснения причин требуются дальнейшие исследования с помощью электронной микроскопии.

Мы исследовали также спектры поляризованной фотолюминесценции „лежащей“ нанонити. Чтобы сделать это, одну нанонить „отрезали“ от подложки и помещали на специально подготовленный трафарет из кремния.

Спектры фотолюминесценции ,лежащей“ нанонити регистрировались с помощью микроскопа и спектрометра, оснащенного CCD-детектором. Пространственное разрешение установки составляло примерно $1.5 \mu \mathrm{m}$. Поскольку размер нити был намного меньше длины волны света и разрешения устройства, в оптическом микроскопе она выглядит как светящаяся точка. Поэтому нам не удалось идентифицировать направление поляризации в соответствии с направлением оси нанонити. Мы измерили всю картину поляризации во всех направлениях. Эта диаграмма направленности показана на рис. 4.

На этом рисунке видно, что излучение экситона в квантовой точке из боковой поверхности нанонити не имеет ярко выраженной линейной поляризации. Если бы экситон в нашей структуре был бы квазидвумерным, т.е. его диаметр был бы больше толщины вдоль оси нити, то в силу правил отбора экситонного перехода излучение было бы на $100 \%$ линейно поляризовано перпендикулярно оси нити [13]. Компонента излучения, поляризованная вдоль оси проволоки, при аннигиляции экситона с тяжелой дыркой в КТ должна отсутствовать. Однако в данных спектрах все наблюдаемые линии слабо поляризованы или не поляризованы вообще.

Для объяснения этого явления следует предположить, что основным состоянием экситона в данной КТ явля- ется состояние экситона с легкой дыркой. Для легкого экситона имеются компоненты дипольного момента как в направлении оси нити, так и перпендикулярно оси.

Геометрически отношение толщины и диаметра КТ достигает 10 и, следовательно, КТ должна была бы быть похожа по своим свойствам на квантовую яму. Однако из-за дополнительного диэлектрического конфайнмента в плоскости, перпендикулярной нити, и недостаточно большой высоты барьеров на границе точки и нити вдоль оси нити основным состоянием экситона становится экситон с легкой дыркой [14]. В результате излучение из боковой поверхности нити оказывается не поляризованным. Наличие слабой, или полное отсутствие линейной поляризации, связано с параметрами нити, в частности с ее диаметром. Мы считаем, что на нитях большого диаметра линейная поляризация излучения из боковой поверхности есть, а на нитях малого диаметра она отсутствует. Это связано с формой волновой функции экситона. Он либо находится внутри точки, как квазидвумерный, и тогда поляризация есть. Либо точка является всего лишь слабым локализующим потенциалом, а экситон является квазиодномерным, и тогда поляризации нет.

\section{3. Заключение}

В спектрах поляризованной фотолюминесценции одиночной квантовой точки, заключенной в нанонить, наблюдались два удивительных явления. В тех случаях, когда не ожидалось никакой линейной поляризации излучения (и не наблюдалось [15]) при рекомбинации экситона, находящегося в основном состоянии, наблюдалась значительная линейная поляризация излучения, достигающая 50\%; а в тех случаях, когда ожидалась $100 \%$-ная линейная поляризация (и наблюдалась в [15]), 
наблюдалась очень слабая линейная поляризация или поляризация вообще отсутствовала.

Первое явление - наличие линейной поляризации, там где ее не должно быть, объясняется анизотропией оптических свойств, вызванной либо искажением формы нити, либо не аксиальным расположением квантовой точки в нанонити. В условиях сильного диэлектрического контраста анизотропное диэлектрическое усиление взаимодействия экситона со светом может приводить к появлению заметной анизотропии в плоскости, перпендикулярной оси нити.

Второе явление - отсутствие поляризации, там где ее было бы естественно ожидать. В этом случае не наблюдалось никакой линейной поляризации ФЛ или наблюдалась очень слабая поляризация. Это объясняется тем, что в условиях дополнительного диэлектрического конфайнмента, перпендикулярного оси нити, основным состоянием экситона в КТ становится экситон с легкой дыркой.

\section{Список литературы}

[1] V.N. Kats, V.P. Kochereshko, A.V. Platonov, T.V. Chizhova, G.E. Cirlin, A.D. Bouravleuv, Yu.B. Samsonenko, I.P. Soshnikov, E.V. Ubyivovk, J. Bleuse, H. Mariette. Semicond. Sci. Technol. 27, 015009 (2012).

[2] D. Barettin, A.V. Platonov, A. Pecchia, V.N. Kats, G. E. Cirlin, A.D. Bouravleuv, L. Besombes, H. Mariette, M. Auf Der Maur, A. Di Carlo. IEEE J. Select. Top. Quantum Electron. 19, 1901209 (2013).

[3] В.Г. Дубровский. Теоретические основы технологии полупроводниковых наноструктур. Изд-во СПбГУ, СПб (2006). $343 \mathrm{c}$.

[4] А.В. Платонов, В.П. Кочерешко, В.Н. Кац, Г.Э. Цырлин, А.Д. Буравлев, Ю.Б. Самсоненко, L. Besombes, H. Mariette. Поверхность 7, 20 (2013).

[5] M.J. Snelling, E. Blackwood, C.J. McDonagh, R.T. Harley, C.T.B. Foxon. Phys. Rev. B 45, 3922(R) (1992).

[6] D.C. Reynolds. Phys. Rev. B 35, 4515 (1987).

[7] Л.Д. Ландау, Е.М. Лифшиц. Квантовая механика. Наука. М. (1974).

[8] L.C. Smith, J.J. Davies, D. Wolverson, S. Gramplin, R.T. Cox, J. Cibert, H. Mariette, V.P. Kochereshko, M. Wiater, G. Karczewski, T. Wojtowicz. Phys. Rev. B 78, 085204 (2008).

[9] Л.В. Келдыш. Письма в ЖЭТФ 29, 716 (1979).

[10] Е.А. Андрюшин, А.П. Силин. ФТП 27, 1256 (1993).

[11] Е.А. Муляров, С.Г. Тиходеев. ЖЭТФ 111, 274 (1997).

[12] D. Gammon, E.S. Snow, B.V. Shanabrook, D.S. Katzer, D. Park. Phys. Rev. Lett. 76, 3005 (1996).

[13] M.H.M. van Weert, N. Akopian, F. Kelkensberg, U. Perinetti, M.P. van Kouwen, J. Gómez Rivas, M.T. Borgström, R.E. Algra, M.A. Verheijen, E.P.A.M. Bakkers, L.P. Kouwenhiven, V. Zwiller. arXiv:0808.2908[cond-mat.mes-hall].

[14] М.А. Семина, Р.А. Сурис. ФТП 7, 947 (2011).

[15] G. Bulgarini, M.E. Reimer, V. Zwiller. Appl. Phes. Lett. 101, 111112 (2012)

Редактор Ю.Э. Китаев 